\title{
Intervención con Jóvenes NiNi en Centros de Inserción Socio Laboral: Mejora de la Autoestima y el Miedo a la Evaluación Negativa
}

\section{The Assistance to NEET Youths in Work Insertion Centers: Development of Self-Esteem and Fear of Negative Evaluation}

\author{
Ana Cristina Ruiz-Mosquera ${ }^{1, \star}, \mathrm{M}^{\mathrm{a}}$ Josefa Vázquez-Fernández ${ }^{2}$, Evaristo Barrera-Algarín ${ }^{2}$ \\ ${ }^{1}$ Universidad de Málaga, España \\ ${ }^{2}$ Universidad Pablo de Olavide, España
}

\begin{abstract}
DESCRIPTORES:
Centros de inserción socio laboral

Jóvenes; NINI

Autoestima

Miedo a la evaluación negativa

RESUMEN:

Se presenta investigación con jóvenes NiNi para conocer cómo influye en ellos el paso por un centro de inserción socio laboral. Hipótesis de investigación: Los programas de los centros de inserción socio laboral de jóvenes NiNi son capaces de fortalecer la autoestima y reducir el miedo a la evaluación negativa. Metodología: Investigación experimental tipo Posttest-Only Control Group Design, con 2 grupos control a 34 sujetos que quedan ubicados en 3 grupos: Grupo Experimento ( 9 meses y más en programas de inserción socio laboral), grupo control A (hasta 2 meses en programas de inserción socio laboral), y grupo control B (entre 3-8 meses). Se aplica cuestionario de variables socio demográficas (Alfa de Crombach), Escala de Autoestima de Rosenberg, y Escala de Miedo a la Evaluación Negativa (FNES). Se realiza análisis de frecuencias, análisis de correlaciones, de fiabilidad y prueba de U de Mann-Whitney. Resultados: Los jóvenes NINI entran en programas de inserción socio laboral buscando aspectos de mejora en su vida; Se produce una mejora de la autoestima y reduce su miedo a la evaluación negativa; factores correlacionados $(p<0,001)$. A más tiempo en los programas mayor implicación de los jóvenes en términos de asiduidad y asistencia a los mismos.
\end{abstract}

\section{KEYWORDS:}

Work insertion centers

Youths; NEET

Self-esteem

Fear of negative

evaluation

\section{ABSTRACT:}

A research study is carried out with NEET young people to find out how a visit to a labour insertion centre can influence. Hypothesis: The programs of work insertion centers of NEET young people are able to strengthen self-esteem and reduce the fear of negative evaluation. The programs of work insertion centers of NEET young people are able to strengthen self-esteem and reduce the fear of negative evaluation. Methodology: Experimental research type Posttest-Only Control Group Design, with 2 control groups, with 34 subjects that are placed in 3 groups: Experimental group ( 9 months and more in programs of work insertion centers), and control group B (between 3-8 months). A survey of socio-demographic variables is applied, Rosenberg's Self-Esteem Scale, and Fear of Negative Evaluation Scale (FNES), through individualized interviews. Frequency analysis, correlation analysis, reliability analysis and Mann-Whitney U test are applied. Results: NEET youths access to social and work insertion programs looking for aspects of improvement in their lives. There is an improvement in self-esteem and a reduction in their fear of negative evaluation; Both factors are also correlated $(p<0,001)$. More time in the programs is spent involving the youths in terms of assiduity and attendance.

CÓMO CITAR:

Ruiz-Mosquera, A. C., Vázquez-Fernández, M. J. y Barrera-Algarín, E. (2021). Intervención con jóvenes NiNi en centros de inserción socio laboral: Mejora de la autoestima y el miedo a la evaluación negativa. Revista Internacional de Educación para la Justicia Social, 10(1), 167-180. https://doi.org/10.15366/riejs2021.10.1.010

*Contacto: acruizmosquera@uma.es

ISSN: 2254-3139

revistas.uam.es/riejs 


\section{Revisión de la literatura}

En la actualidad no encontramos una definición exacta de NiNi o joven que ni estudia ni trabaja ni se encuentra en prácticas. Se debe considerar un término más que un concepto debido a que en el ámbito internacional no existe consenso acerca de su definición (de forma especial, cuando tratamos de seleccionar la población a quién incluir dependiendo del rango de edad y la condición de actividad del individuo) así lo avalan los estudios de Rennison y otros (2005), Spielhofer y otros (2007) y Edcoms (2009). Se puede afirmar que presenta puntos problemáticos en su definición como también expusieron Negrete y Leyva (2013) al poner en contexto este término. Debido a esta razón, en cada estudio que se lleva a cabo en torno a este eje temático se establece una definición girando principalmente sobre el rango de edad de acuerdo con el interés del mismo, ligado en su totalidad al conjunto de la juventud.

\subsection{Nociones sobre el término NiNi}

A nivel internacional la Organización de Naciones Unidas (2014), por ejemplo, define a este sector de la población como aquellas personas entre las edades de 15 a 24 años que ni estudian ni trabajan, utilizando esta forma de cuantificarlos persiguiendo fines estadísticos; la Organización de Cooperación para el Desarrollo Económico (2014), por otro lado, difunde sus estadísticas sobre este colectivo haciendo referencia a la población de edades comprendidas entre los 15 y los 29 años mientras que la Organización Internacional del Trabajo (2015) define las siglas NEET como los jóvenes que no tienen empleo, educación o formación, las personas de 15 a 24 años que están desempleadas y fuera del sistema educativo, estableciendo por lo tanto el mismo rango de edad que la Organización de Naciones Unidas incluyendo eso sí la actividad de formación en prácticas.

En la actualidad, en España encontramos un término de NiNi más complejo y amplio, que recoge los distintos perfiles de jóvenes que se encuentran sin estudiar ni trabajar. De acuerdo con Ruiz, Palma y Álvarez (2019) los jóvenes NiNi son aquellos que están sin formar parte del sistema educativo ni del mercado laboral y se pueden clasificar en: NEET vocacionales (aquellos que eligen estar en esa situación), NEET resultado del sistema educativo (respondiendo a este perfil los jóvenes que abandonan sus estudios en las distintas etapas del recorrido académico) y los jóvenes NEET excluidos del mercado laboral (ya sea por sobrecualificación, subcualificación o no ser los elegidos en las entrevistas de trabajo por presentar carencia de experiencia profesional).

Cuadro 1

Evolución número de jóvenes NINI en España entre los años 2013-2019

\begin{tabular}{cccccccc}
\hline Año & $\mathbf{2 0 1 3}$ & $\mathbf{2 0 1 4}$ & $\mathbf{2 0 1 5}$ & $\mathbf{2 0 1 6}$ & $\mathbf{2 0 1 7}$ & $\mathbf{2 0 1 8}$ & $\mathbf{2 0 1 9}$ \\
\hline$\%$ NINI & $22,5 \%$ & $20,7 \%$ & $19,4 \%$ & $18,1 \%$ & $16,4 \%$ & $15,3 \%$ & $12,8 \%$ \\
\hline
\end{tabular}

Nota. Elaboración propia a partir del INE (2020).

Como muestra la tabla anterior, cabe destacar el marcado descenso de jóvenes NiNi en los últimos siete años (9,7\%), de tal modo que, si en el año 2013 alcanzó un 22,5\%, según el INE (2020), inicia un descenso progresivo anual, hasta llegar al 12,8\% en 2019.

\subsection{Factores relacionados con la situación de NiNi}

Bivand (2004), describe que factores, como las expectativas familiares o culturales, los sistemas de creencias de los jóvenes, las relaciones sociales con los compañeros (incluso el no gustarle la perspectiva de trabajo o asistir a un curso que realmente no les suscita interés) y el atractivo que ciertos estilos de vida representan, entre otros, pueden explicar la situación en la que se encuentran los jóvenes NiNi. De estas investigaciones se deduce entre líneas que, con frecuencia, los jóvenes no siempre se convierten en NiNi como resultado de situaciones de desventaja, sino que en algunos casos, puede existir un elemento activo de "elección".

En lo que respecta a la relación de los jóvenes NiNi con la propia familia, cabe destacar que, al igual que sucede con el resto de jóvenes, en la etapa de la adolescencia, se suelen romper vínculos con los padres, puesto que, con la pubertad, se inaugura una etapa transitiva hacia la edad adulta, en la cual, acontecen, además de los cambios físicos, otros cognitivos, apareciendo así una menor sintonía con los padres y mayor identificación con el grupo de iguales o de amigos. En este sentido, se sienten menos comprendidos por sus progenitores a la vez que más identificados con el grupo de iguales, puesto que este, constituye un nuevo espacio de referencia 
afectiva y social. De este modo, son numerosos los padres que, con frecuencia, dicen sentirse desplazados, en el sentido de que, constatan su pérdida de influencia sobre los hijos, frente al grupo de amigos, al que perciben como una especie de contrincante o rival.

Los jóvenes NiNi, por su parte, suelen relacionarse con amigos coincidentes con su misma escala de valores y aspiraciones, lo cual no siempre es motivador para realizar cambios positivos, teniendo en cuenta que el grupo de iguales, ejerce una relevante influencia en aspectos morales, académicos y hasta de salud, según el nivel de autoestima y seguridad personal.

No cabe duda que, junto con los cambios en los hijos adolescentes, aparece para los padres, no solo una etapa de transformación sino también de preocupación, exigiéndoles reformulaciones conceptuales y readaptaciones a nivel familiar, teniendo que afrontar la pérdida de ascendiente e influencia, dando cabida a nuevos comportamientos y aspiraciones del adolescente. En definitiva, los padres de los jóvenes que nos ocupan, describen sentirse con frecuencia, desconcertados.

\subsection{Autoestima y Motivación por el cambio de situación NiNi}

En lo que respecta a la motivación de estos jóvenes para participar en el tipo de programas formativos que veremos más adelante, es evidente que tras una etapa, con frecuencia prolongada, en la que no sienten motivación para reanudar estudios o incorporarse al mercado laboral, según los profesionales de dicho programas, consultados para el trabajo que nos ocupa, es frecuente que, en un determinado momento de sus vidas, pueden acontecer circunstancias de diversa índole conducentes a un cambio de actitud positiva y de apertura hacia algún tipo de meta y de horizonte. Con frecuencia, son otros jóvenes del entorno social al que pertenecen que, incorporados ya a un programa de formación, les transmiten su experiencia positiva, sus ilusiones y las ventajas prácticas que tiene para su vida (mayor accesibilidad a oportunidades formativas y de inserción laboral, y, por consiguiente, mejores condiciones de vida para el futuro, con lo cual, despierta el interés y la motivación de otros. También, en ocasiones, surge un cambio de actitud y motivación, gracias al acompañamiento llevado a cabo por trabajadores sociales, psicólogos, educadores de calle y animadores socioculturales.

En lo que se refiere a programas existentes para la formación e inserción de jóvenes NiNi en España, no existen datos publicados por parte del Ministerio del Interior referentes a entidades con este cometido, si bien se han podido localizar aproximadamente 200 entidades, cuya labor se orienta a la formación e inserción socio laboral de jóvenes sin cualificación, que no estudian ni trabajan o en riesgo de exclusión social.

Para Manassero y Vázquez (1995), la baja autoestima queda vinculada a muchos fracasos académicos, relacinados de forma directa con la tendencia a que esa persona desarrolle su día a día sin estudiar ni trabajar, influyendo de forma directa con el bienestar subjetivo descrito por Murillo y Salazar (2019). A través de la literatura (Brooks, 1998; Edcoms, 2007; Steers, 2000) se corrobora que existe un fuerte vínculo entre el bajo rendimiento académico y la probabilidad de que un joven se convierta en NiNi. Por lo tanto, es importante conocer el nivel de autoestima en la población de estudio relacionada con el rendimiento académico con la finalidad de poder diseñar itinerarios de inserción sociolaboral que permitan mejorar la autoestima de los jóvenes estudiantes de ser el caso favoreciendo su reinserción en el sistema educativo o en el mercado laboral y por ende en la sociedad.

La autoestima supone la evaluación global de la consideración positiva o negativa de la propia persona. Es un componente del más amplio concepto de uno mismo, que Rosenberg (1989) la vincula a la totalidad de los pensamientos y sentimientos de la persona con referencia a sí mismo como objeto. Además de la autoestima, la autoeficacia y la autoidentidad son una parte importante del concepto de sí mismo, para el que construyó un inventario de escala de autoestima.

Acuña (2013), en el contexto de investigaciones realizadas para evaluar la relación de la autoestima con el rendimiento académico, encontró que existe una correlación positiva entre la autoestima y el rendimiento académico de los estudiantes. Los jóvenes que presentan elevadas percepciones de competencia, al contrario de lo que ocurre con aquellos que tienen una autopercepción de poco eficaces, manifiestan curiosidad e interés por el aprendizaje (Díaz-Rodríguez, 2020).

A través de la enseñanza, y concretamente desde la intervención social, se promueve el incremento de la autoestima, el autoconocimiento, el autoconcepto, se promociona la motivación por los estudios y la profesión y a su vez, la futura inserción socio-laboral de estos estudiantes (Ruiz-Mosquera, 2020). Para Bolívar (2006), la 
autoestima que desarrollan las personas en su vida diaria, ya sean constructivas o destructivas influyen en la formación de su propio autoconcepto y por lo tanto en la evaluación que hace cada persona de sí mismo. Para poder atender a las críticas, la evaluación negativa (ya sea intrapersonal o social) la persona tiene que tener seguridad de sí mismo para poder discernir entre qué crítica va a favor y cual en contra de su integridad como persona, debido a que una crítica negativa es capaz de devaluar a una persona y de afectar en gran medida al amor propio, llevándolo incluso a sentirse que es un verdadero fracaso, ya sea en el ámbito escolar o laboral en su faceta personal o socialmente.

Las referencias a factores de actitud o de comportamiento en relación al fenómeno NiNi han sido ampliamente citadas a través de la literatura. Ejemplo de ello son Sims y otros (2001) y Golden y otros (2008), que informan de que los jóvenes con los que han realizado sus estudios a menudo presentan gran diversidad de problemas personales que se reflejan en su actividad. Cuando se refieren a problemas personales están haciendo mención a baja confianza o autoestima, enfados, baja o nula motivación, dificultad en las relaciones con profesores o compañeros lo que provoca la percepción de una evaluación negativa por parte de las personas que los rodean. En otros casos, también se hace referencia a que los jóvenes simplemente carecen de confianza o de las habilidades personales a la hora de continuar formando parte del sistema educativo o durante el tránsito al mercado laboral.

\subsection{Nivel educativo y la tendencia a no estudiar ni trabajar}

Department for Children, Schools and Families (2008) señaló con su publicación estadística, por ejemplo, que aquellos jóvenes que no superaron los estudios básicos fueron registrados como NiNis posteriormente. Además, Fergusson (2004), en una encuesta realizada a más de 800 estudiantes de entre 16 y 18 años de edad, descubrió que los jóvenes que tenían niveles de logros educativos más bajos presentaban menos probabilidad de un desarrollo de futuro efectivo una vez superados los 16 años que aquellos que obtuvieron buenos resultados académicos. Es decir, aquellos con dificultades en el desarrollo académico presentaban mayor tendencia a atravesar periodos de vacío en cuanto a estudios y trabajo.

Por su parte, Cartmel (2000) señaló que tenían una mayor tendencia a ser desempleados aquellos jóvenes de entre 16 y 17 años que no habrían realizado una transición positiva durante el periodo educativo que aquellos jóvenes que si protagonizaron una transición correcta conforme al diseño del sistema educativo. Estos jóvenes sin formación son los que la literatura española, Morales (2015) califica como auténticos NEET, jóvenes no empleables por su falta, baja o inadecuada formación, dirigiendo la atención a que esa falta de formación nace como resultado de nuestras altas tasas de fracaso y posterior abandono escolar.

La desconexión, el absentismo y el bajo rendimiento se sustentan por la compleja interacción de múltiples y diversos factores, que se extienden a los ámbitos social, cultural, educativo y otras áreas de la vida de estos jóvenes como concluyen Archer y otros (2005). Ruiz-Mosquera y Arredondo (2020) sintetizan como esencial la labor preventiva sobre la intervención con estos jóvenes, es decir, tratar de reducir y/o eliminar los factores que de manera directa o indirecta afectan a que se generen estos procesos. Sin dejar de resaltar que sería inexacto sugerir que todos los jóvenes que están en situación de NiNi se enfrentan a las mismas circunstancias, o que atraviesan similares situaciones de desventaja. Dicho de otra forma, los jóvenes pueden sufrir una combinación de factores incluyendo los mencionados anteriormente: baja autoestima, miedo a la evaluación negativa, bajo logro académico, antecedentes familiares complicados, atravesar por dificultades personales y, quizás como resultado, mostrar actitudes desafiantes o comportamientos que denoten una evaluación negativa que Stone y otros (2000) describen dentro de un 'efecto espiral excluyente'. La propia experiencia nos permite afirmar que para poder elaborar un plan de intervención adecuado y acorde al perfil de cada persona es imprescindible conocer y analizar en profundidad la situación de cada uno en particular (Ruiz-Mosquera y Vázquez-Fernández, 2020).

A partir de aquí nos planteamos la siguiente hipótesis de investigación: Los programas de los centros de inserción sociolaboral de jóvenes NiNi (que ni estudiaban ni trabajaban) son capaces de fortalecer la autoestima y reducir el miedo a la evaluación negativa. Y con ello formulamos el objetivo del estudio: Realizar estudio de investigación con jóvenes NiNi para conocer cómo el paso por un centro de inserción sociolaboral puede influir en su autoestima y su miedo a la evaluación negativa. 


\section{Método}

\section{Tipo de investigación}

Se trata de una investigación experimental del tipo Posttest-Only Control Group Design, con 2 grupos control. En los 3 grupos se administran medidas de observación post-test, con los instrumentos que hemos descrito. Se utiliza este tipo de investigación experimental porque, como indican Rubin y Babbie (2005), es aconsejable cuando, pudiendo realizar asignación aleatoria de la muestra a la conformación de los grupos experimental y de control, no es posible realizar una observación pre-test, y lo que se pretende es medir los efectos de algún tipo de acción, programa o actividad anterior.

\section{Proceso de investigación}

Los cuestionarios se llevaron a cabo durante junio y julio de 2019. Los lugares elegidos para su paso fueron el Centro Don Bosco situado en las Tres Mil Viviendas (Sevilla) y en el barrio de La Trinidad (Málaga) y la duración fue de una hora y media.

En todos los casos, tras invitar a los participantes a formar parte de la investigación se ha informado sobre la voluntariedad y finalidad de la misma, garantizando la confidencialidad de los datos recabados. El estudio se ha realizado conforme al Código de Ética de Trabajo Social y de la Federación Internacional de Trabajadores Sociales, y a la Ley Orgánica 3/2018, de 5 de diciembre, de Protección de Datos Personales y garantía de los derechos digitales.

\section{Instrumentos}

Inicialmente se trabaja con tres instrumentos: Un cuestionario auto confeccionado de análisis de variables sociodemográficas, una escala de autoestima, y una escala de miedo a la evaluación negativa.

Variables sociodemográficas: Instrumento con el objeto de recoger las variables sociodemográficas de interés para la investigación. Las variables recogidas en este instrumento son: Centro de inserción, edad, sexo, estado civil, hijos, y nivel de estudios, edad de abandono de los estudios, cursos finalizados, curso de abandono de los estudios, asiduidad de asistencia al centro, grupo de pertenencia, tiempo formándose en el centro, motivos por los que decide entrar en los programas de inserción socio laboral. El instrumento arroja una fiabilidad de Alfa de Cronbach de r=0,776.

Escala de Autoestima de Rosenberg (Rosenberg, 1965), en su versión española adaptada de Vázquez, Jiménez y Vázquez-Morejón (2004). Escala para explorar la autoestima personal entendida como los sentimientos de valía personal y de respeto a sí mismo. La escala consta de 10 ítems con los que se consigue catalogar al sujeto en: Autoestima elevada (considerada como autoestima normal), Autoestima media (no presenta problemas de autoestima graves, pero es conveniente mejorarla) y Autoestima baja (donde existen problemas significativos de autoestima). La consistencia interna de las escalas se encuentra entre 0,76 y 0,87. La fiabilidad es de $r=0,800$.

Escala de Miedo a la Evaluación Negativa (Feer of Negative Evaluation Scale, FNES) de Watson y Friend, 1969, en su versión validada al español de Zubeidat, Salinas y Sierra (2007). El instrumento mide la intensidad del temor experimentado por los sujetos ante la posible evaluación negativa por parte de los demás. Escala de 30 ítems de verdadero/falso, que a partir de un rango de puntos de entre 0-30, permiten agrupar al sujeto en 3 grupos: Bajo miedo a la evaluación negativa, moderado miedo a la evaluación negativa y elevado miedo a la evaluación negativa. La fiabilidad de la escala es de $r=0,850$.

\section{Participantes del estudio}

La selección muestral parte de un universo de 105 jóvenes que participaron en los programas de formación en el año 2019, en los centros de inserción sociolaboral Don Bosco, situados en el Polígono Sur de Sevilla y en el barrio de La Trinidad de Málaga. Sobre este universo, aplicamos los siguientes criterios de inclusión: Estar participando en programas de inserción socio laboral de alguna de las 2 instituciones; no estar estudiando ni trabajando en el momento en que se quiso acceder a los programas del centro; tener edad comprendida entre 13 y 25 años; tener autorización de los tutores legales, del centro o del propio sujeto en caso de ser mayor de edad. Criterios de exclusión: Estar trabajando o estudiando en el momento de entrada a los programas de las instituciones; ser mayor de 25 años o menor de 13 años; presentar alguna limitación que le impida contestar a las distintas escalas y cuestionario. Tras la aplicación de estos criterios, se determina una muestra inicial de 62 sujetos, a los que se aplica fórmula de aleatoriedad de Excel. Finalmente se obtiene una muestra definitiva de 34 sujetos (ver tabla 2), 
que quedan ubicados en 3 grupos: Grupo Experimento (sujetos que llevan más de 9 meses en programas de inserción socio laboral), grupo control A (sujetos que llevan entre 2-9 meses en programas de inserción socio laboral), y grupo control B (A (sujetos que llevan hasta 2 meses en programas de inserción socio laboral). Es importante entender que la distinción en un Grupo Experimental y dos grupos control ("A" $\mathrm{y}$ “B”) nos permiten, de un lado, tener un mayor control sobre las variables del grupo experimental al poderlo comparar con dos grupos control, y de otro, medir la sensibilidad y vinculación con la variable "tiempo en el centro" (tiempo de tratamiento).

Cuadro 2

Género, Edad, Estado Civily Centro de Inserción Según Grupo Muestral

\begin{tabular}{|c|c|c|c|c|c|c|c|c|c|c|c|}
\hline & & & & \multicolumn{2}{|c|}{ Hombre } & \multicolumn{4}{|c|}{ Mujer } & \multicolumn{2}{|r|}{ Total } \\
\hline \multicolumn{12}{|l|}{ Género } \\
\hline Grupo A (hasta dos meses) & & & & \multicolumn{2}{|c|}{8} & \multicolumn{4}{|c|}{2} & \multicolumn{2}{|r|}{10} \\
\hline Grupo B (entre 3-8 meses) & & & & \multicolumn{2}{|c|}{9} & \multicolumn{4}{|c|}{3} & \multicolumn{2}{|r|}{12} \\
\hline Grupo Experimento ( 9 mese & $\mathrm{y} \mathrm{m}$ & & & \multicolumn{2}{|c|}{5} & \multicolumn{4}{|c|}{7} & \multicolumn{2}{|r|}{12} \\
\hline Total & & & & \multicolumn{2}{|c|}{22} & \multicolumn{4}{|c|}{12} & \multicolumn{2}{|r|}{34} \\
\hline Edad & 13 & 15 & 16 & 17 & 18 & 19 & 20 & 21 & 22 & 25 & Total \\
\hline Grupo A (hasta dos meses) & 0 & 0 & 0 & 3 & 3 & 2 & 1 & 0 & 0 & 1 & 10 \\
\hline Grupo B (entre 3-8 meses) & 0 & 0 & 1 & 3 & 6 & 1 & 0 & 1 & 0 & 0 & 12 \\
\hline $\begin{array}{l}\text { Grupo Experimento ( } 9 \\
\text { meses y más) }\end{array}$ & 1 & 1 & 3 & 1 & 2 & 2 & 1 & 0 & 1 & 0 & 12 \\
\hline Total & 1 & 1 & 4 & 7 & 11 & 5 & 2 & 1 & 1 & 1 & 34 \\
\hline \multicolumn{4}{|l|}{ Estado civil } & \multicolumn{2}{|c|}{ Soltero/a } & \multicolumn{4}{|c|}{ Pareja de hecho } & \multicolumn{2}{|r|}{ Total } \\
\hline \multicolumn{4}{|l|}{ Grupo A (hasta dos meses) } & \multicolumn{2}{|c|}{9} & \multicolumn{4}{|c|}{1} & \multicolumn{2}{|r|}{10} \\
\hline \multicolumn{3}{|l|}{ Grupo B (entre 3-8 meses) } & & \multicolumn{2}{|c|}{11} & \multicolumn{4}{|c|}{1} & \multicolumn{2}{|r|}{12} \\
\hline \multicolumn{3}{|c|}{ Grupo Experimento (9 meses y más) } & & \multicolumn{2}{|c|}{11} & \multicolumn{4}{|c|}{1} & \multicolumn{2}{|r|}{12} \\
\hline \multicolumn{3}{|l|}{ Total } & & & & & & 3 & & & 34 \\
\hline Centro de inserción & & & & illa & & & & & ga & & Total \\
\hline Grupo A (hasta dos meses) & & & & 9 & & & & & & & 10 \\
\hline Grupo B (entre 3-8 meses) & & & & 7 & & & & & & & 12 \\
\hline $\begin{array}{l}\text { Grupo Experimento ( } 9 \\
\text { meses y más) }\end{array}$ & & & & & & & & & & & 12 \\
\hline Total & & & & 18 & & & & & & & 34 \\
\hline
\end{tabular}

Nota. Elaboración propia.

Del total de sujetos (34) al grupo experimento pertenecen 12, al grupo A, 10 y al grupo B, 12, quedando una muestra bien proporcionada. En términos generales son 16 sujetos seleccionados aleatoriamente en el Centro de Málaga, y 18 en el de Sevilla. Mayoritariamente son sujetos solteros (91,2\%), donde la franja de edad predominante se sitúa entre los 16 a 19 años (79,5\% de la muestra), y hay más hombres (65\%) que mujeres (35\%).

En relación al perfil como estudiantes que presenta la muestra, podemos destacar que, en su mayoría, son sujetos que tienen los estudios primarios (45,5\%), y un 33,4\% ha llegado a iniciar los estudios de Educación Secundaria. Si bien el 12,2\% ha llegado a iniciar estudios de bachiller, también encontramos un 6,1\% de sujetos que presentan menos de 5 años de escolarización. De manera comparada con el alumnado de España, cabe destacar que, teniendo en cuenta los últimos datos publicado de los que se dispone, referidos al curso 2017-2018, el porcentaje de alumnado que no alcanzó la titulación de Graduado en Eso, asciende a 22,2\%, frente al 45,5\% del alumnado que nos ocupa que no pudo alcanzar dicha titulación. Se aprecia también una marcada brecha entre el 100\% de los 
jóvenes españoles que inician la Enseñanza Secundaria y el 33,4\% de los jóvenes de este estudio, que solamente llega a iniciarlos.

\section{Análisis de los datos}

Los datos que se han obtenido, se han trabajado con Excel y se han analizado con el programa informático IBM SPSS Statistics 25. Se aplica análisis de frecuencias, análisis de correlaciones y de fiabilidad (Correlaciones de Pearson). Al tratarse de grupos de menos de 30 sujetos, para la aceptación o rechazo de la hipótesis nula (HO), se ha utilizado la prueba no paramétrica U de Mann-Whitney.

\section{Resultados}

\subsection{Abandono escolar}

En referencia a los datos de abandono escolar (figura 1), el 100\% de la muestra se trata de sujetos, que en el momento de iniciarse en los programas de inserción socio laboral, habían dejado de estudiar. Lo habían hecho en edades entre los 15-17 años, donde se concentra el 70,97\% de la muestra. Estos abandonos se producen en los cursos de Educación Secundaria Obligatoria (ESO) de la siguiente forma: $1^{\circ}$ un $25 \% ; 2^{\circ}$ un $39 \%$; $3^{\circ}$ un $25 \%$; y $4^{\circ}$ un 20\%. En cuanto a España, no han sido publicados datos referidos al porcentaje de jóvenes que abandonan antes de los 16 años, edad hasta la que es obligatoria la educación secundaria de primera etapa, ni los cursos en los que abandonan, si bien los datos publicados, denominan abandono escolar temprano, el abandono que se da entre los 18-24 años, a pesar de que tal y como demuestra la realidad de los jóvenes que nos ocupan, existen alumnos que abandonan el sistema educativo antes de esa edad, incluso previo a los 16 años, a pesar de su obligatoriedad hasta entonces. En el año 2018, el porcentaje de abandono escolar ascendió a 17,9\% cifran notablemente inferior a las que encontramos en los sujetos del estudio.

\section{Figura 1}

Curso y edad de abandono de los estudios
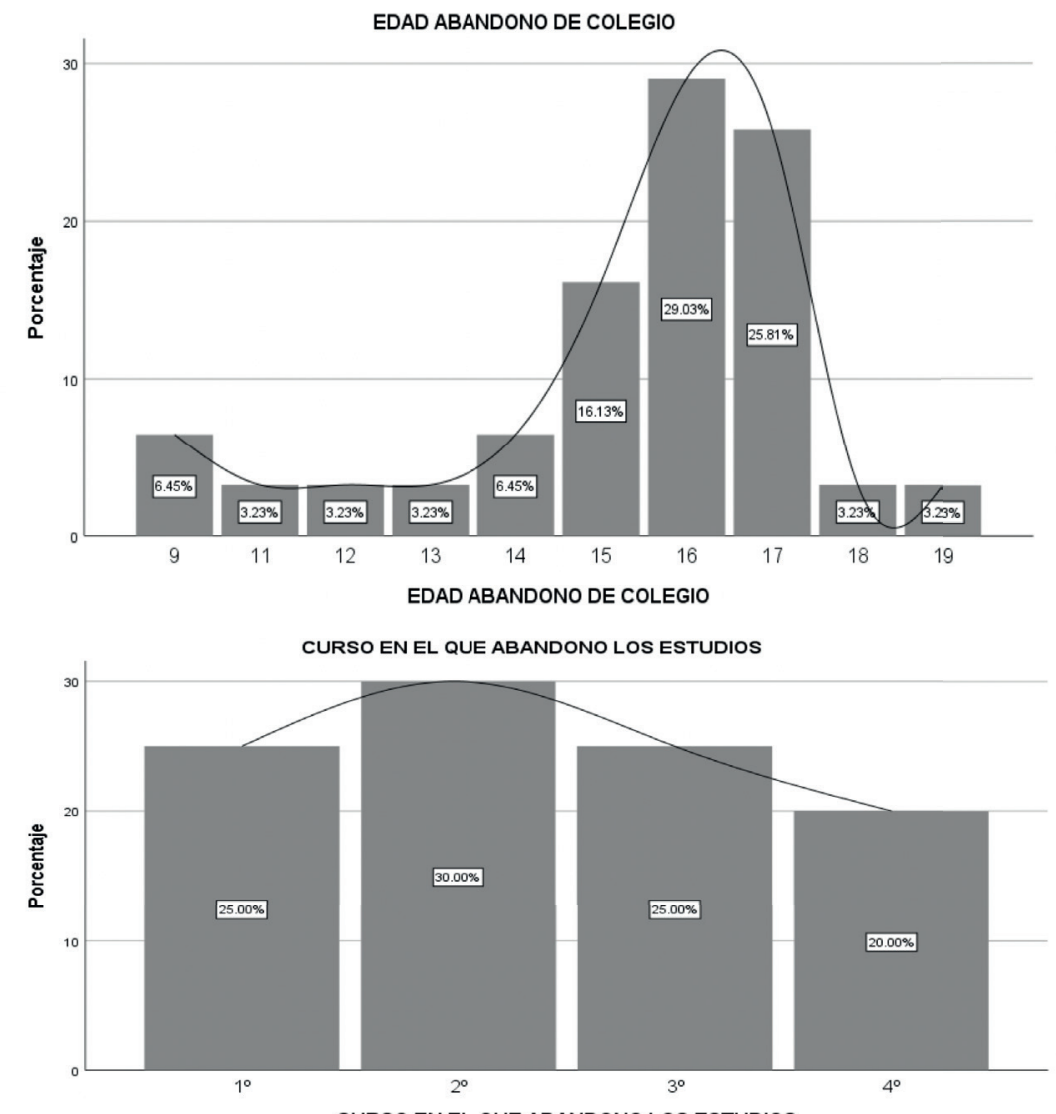
Analizando los datos en referencia al perfil como integrantes en los programas de inserción socio laboral, estos sujetos afirman haber accedido a estos por las siguientes cuestiones de manera mayoritaria: Porque quieren "mejorar su formación" (67,6 \%); porque quieren "encontrar un trabajo" (67,6\%); y porque "busca mejores condiciones de vida en el futuro" (50,0\%). En menor medida nos indican que han entrado en estos programas: Porque está "desempleado" (23,5\%); porque esta "sin estudiar" $(35,3 \%)$; porque esta "aburrido" $(20,6 \%)$; porque "se sentía mal consigo mismo porque tenía la sensación de no hacer nada (17,6\%); porque "su familia le ha animado" $(32,4 \%)$; y porque "sus amistades le han animado" $(11,8 \%)$.

Son una muestra que se fija mucho más en aspectos de mejora (mejorar formación, encontrar trabajo o mejorar sus condiciones de vida), que en carencias que puedan poseer (desempleado, sin estudiar, sentirse mal consigo mismo...). También observamos que la familia tiene poco peso para que estos jóvenes entren en los programas de inserción socio laboral, pero aún menos, las amistades o grupo de iguales.

\subsection{Vinculación con programas de inserción}

Por otro lado, esta muestra, en referencia a la asiduidad de asistencia a los programas de inserción, se caracterizan por una asistencia regular de "Mas de un día a la semana" en un 64,7\% (los programas suelen ser de 2-3 días/semana). Pero si analizamos la comparativa entre el grupo experimento y los grupos Control A y B (ver figura 2), nos damos cuenta que se produce un cambio de tendencia, y los inscritos en los programas aumentan su asistencia a los mismos, pasando la "asistencia diaria" del $0 \%$ en el Grupo A, al $25 \%$ en el Grupo B, y finalmente al 58,4\% en el grupo experimental.

Figura 2

Cambio en la tendencia de asiduidad a los programas (en \%)

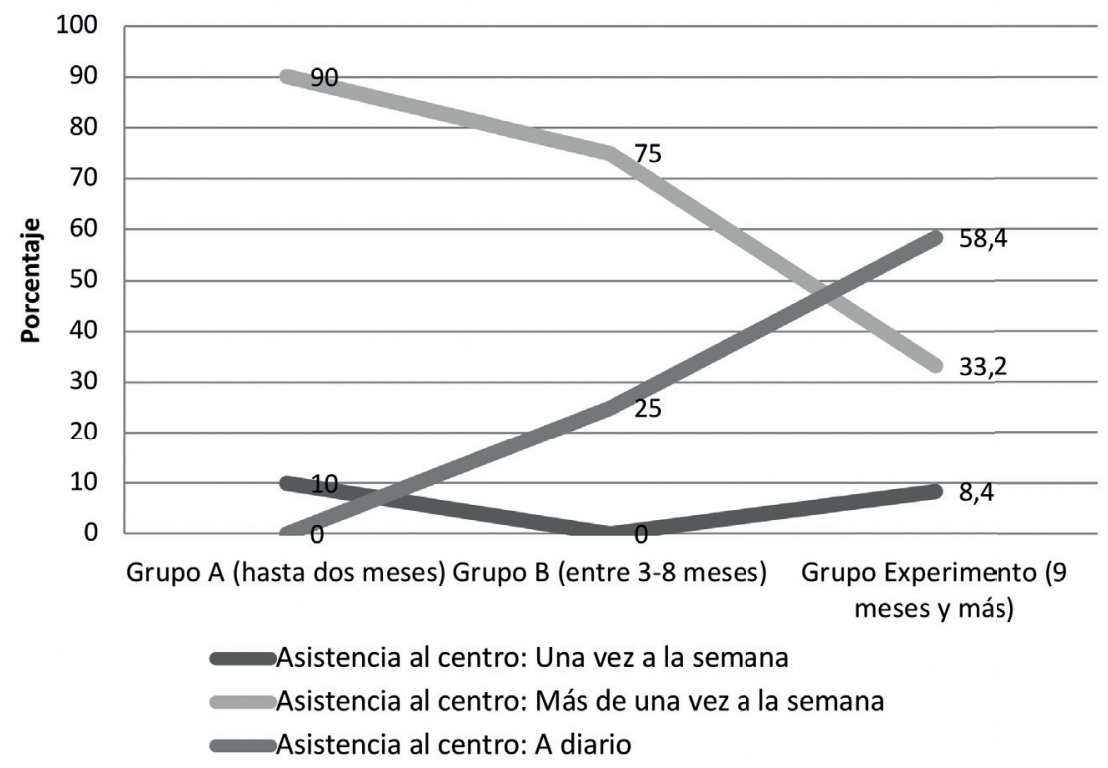

\subsection{Autoestima}

En relación al análisis de la autoestima (a través de la escala Rosenberg), obtenemos como dato general del total de la muestra: Predomina la autoestima "normal" (79,4\%), un 8,8\% presenta una autoestima "alta”, pero de forma preocupante en jóvenes, un 11,8\% presenta una autoestima dañada. Cuando realizamos comparativa entre los grupos podemos apreciar un aumento generalizado de la autoestima (figura 3), cayendo la "Autoestima baja" de un $20 \%$ al $0 \%$ en el grupo experimento, manteniéndose la "autoestima normal", y aumentando la autoestima alta, desde el 10\% en el Grupos A y B, hasta el $20 \%$ en el Grupo Experimento. Con esto vemos confirmada la primera parte de nuestra hipótesis. 
Figura 3

Variación de la autoestima entre los grupos (en \%)

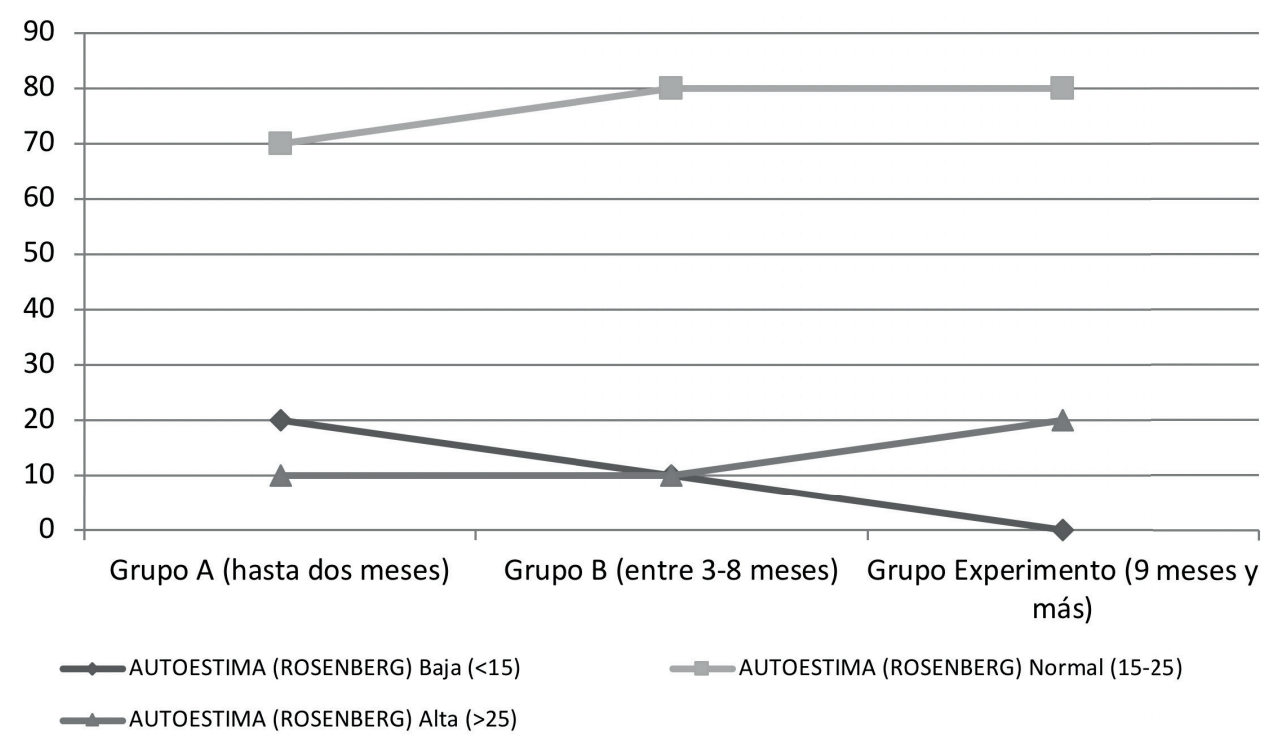

Para ayudar a delimitar aún mejor la confirmación de hipótesis de autoestima, realizamos análisis de U de Mann-Whitney (cuadro 3) que nos da un valor de 1,650 en la comparación entre Grupo control A y Grupo Experimento, siendo el valor de p (Significación asintónica bilateral) de 0,003. Esto significa que se rechaza la hipótesis nula (HO) al estar el valor por debajo de 0,05 (nivel de error en el $5 \%, \alpha=0,05$ ). Es decir, en la comparativa de datos de autoestima se confirma la hipótesis alternativa (H1): Los programas de los centros de inserción socio laboral de jóvenes NiNi (que ni estudiaban ni trabajaban) son capaces de fortalecer la autoestima.

Cuadro 3

U de Mann-Whitney para Rosenberg

\begin{tabular}{ll}
\hline U de Mann-Whitney & 1,650 \\
W de Wilcoxon & 37,500 \\
Z & $-3,867$ \\
Sig. asintótica (bilateral) & 0,003 \\
Significación exacta [2*(sig. unilateral)] & $0,000 \mathrm{~b}$ \\
\hline
\end{tabular}

Nota: a. Variable de agrupación: Grupo de pertenencia. b. No corregido para empates.

\subsection{Miedo a la evaluación negativa}

Cuando analizamos el miedo a la evaluación negativa a través de la Escala de Miedo a la Evaluación $\mathrm{Ne}$ gativa (Feer of Negative Evaluation Scale, FNES), observamos en términos generales, que en la muestra predomina un "bajo miedo a la evaluación negativa" $(52,9 \%)$ y un "moderado miedo a la evaluación negativa" (41,2\%). Pero cuando analizamos las diferencias entre el grupo experimento y los grupos control A y B, obtenemos que: Existe en "elevado miedo a la evaluación negativa " en el Grupo Control A (10\%), que se reduce en el Grupo Control B (8,3), y que desaparece en el Grupo Experimento (los que llevan más tiempo en el programa). También observamos que el "bajo miedo a la evaluación negativa", aumenta, pasando del 50\% en los Grupos Control A y B, hasta el 58,4\% en el Grupo Experimento. Estos datos confirmarían la segunda parte de nuestra hipótesis. 
Figura 4

Variación del miedo a la evaluación negativa entre los grupos (en \%)

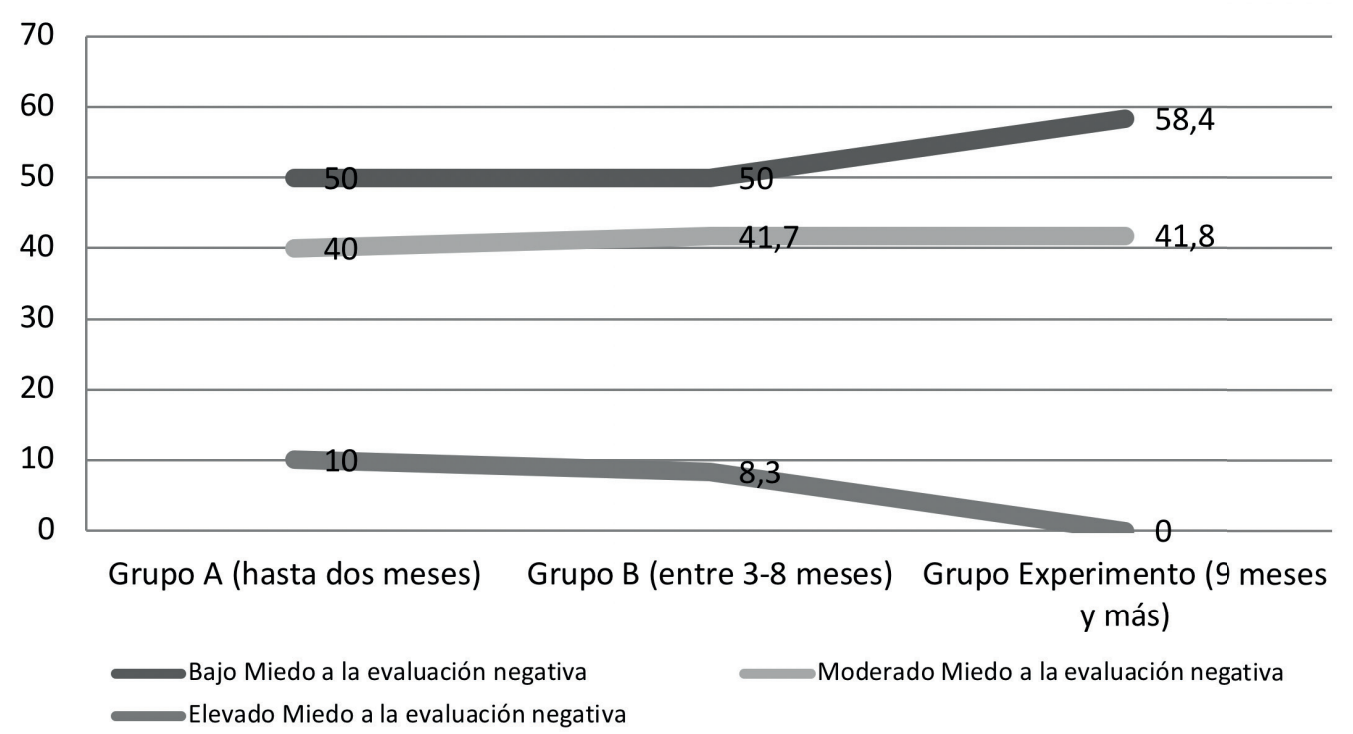

Para mejorar el dato de confirmación de hipótesis sobre el miedo a la evaluación negativa, aplicamos análisis de U de Mann-Whitney (Cuadro 3). La prueba nos da un valor de 2,100 en la comparación entre Grupo control A y Grupo Experimento, siendo el valor de p (Significación asintónica bilateral) de 0,001. Esto significa que se rechaza la hipótesis nula (HO) al estar el valor por debajo de 0,05 (nivel de error en el $5 \%, \alpha=0,05)$. Es decir, en la comparativa de datos de autoestima se confirma la hipótesis alternativa (H1): Los programas de los centros de inserción socio laboral de jóvenes NiNi (que ni estudiaban ni trabajaban) son capaces de reducir el miedo a la evaluación negativa.

Cuadro 3

U de Mann-Whitney para Escala de Miedo a la Evaluación Negativa FNES

\begin{tabular}{ll}
\hline U de Mann-Whitney & 2,100 \\
W de Wilcoxon & 42,600 \\
Z & $-2,729$ \\
Sig. asintótica (bilateral) & 0,001 \\
Significación exacta [2*(sig. unilateral)] & $0,000 \mathrm{~b}$ \\
\hline
\end{tabular}

Nota. a. Variable de agrupación: Grupo de pertenencia. b. No corregido para empates.

\subsection{Autoestima y miedo a la evaluación negativa}

Además, encontramos una correlación entre la evolución positiva de la Autoestima y la reducción del miedo a la evaluación negativa. Aparece una correlación (ver tabla 4) significativa, $\mathrm{x} 2=-0,761, p<0,001$ $(\mathrm{N}=34)$ entre las dos escalas. Estos datos se reflejan también en que el "elevado miedo a la evaluación negativa" sólo está presente en el grupo de jóvenes con la "autoestima baja” (2,9\%), desapareciendo totalmente con los jóvenes que presentan una "autoestima alta". 


\begin{tabular}{llc}
\hline & & Autoestima (Rosenberg) \\
\hline \multirow{2}{*}{$\begin{array}{l}\text { Escala de miedo a la evaluación negativa } \\
\text { (FNES) }\end{array}$} & Correlación de Pearson & $-0,761^{* *}$ \\
& Sig. (bilateral) & 0,001 \\
\hline
\end{tabular}

Nota. ${ }^{* *}$ Correlación significativa.

\section{Discusión}

Tras la finalización del estudio, estamos en disposición de afirmar que hemos alcanzado nuestro objetivo: Realizar estudio de investigación con jóvenes NiNi para conocer cómo el paso por un centro de inserción sociolaboral puede influir en su autoestima y su miedo a la evaluación negativa. A partir de los resultados obtenidos, se concluyen varias cuestiones:

Primera: Entre los jóvenes que han participado en este estudio, destaca el perfil NEET resultado del sistema educativo, ya que han abandonado de forma temprana los estudios y jóvenes NiNi excluidos del mercado de trabajo por no alcanzar la cualificación mínima exigida para realizar trabajos cualificados, circunstancias que los sitúan en ambos casos en riesgo de exclusión social. Coincidimos aquí con las descripciones de Archer y otros (2005), o con las afirmaciones de Fergusson (2004) sobre su relación entre las dificultades en el desarrollo académico, y los periodos de vacío en estudios y trabajo. Y con las aportaciones de Ruíz, Palma y Álvarez (2015) y Morales (2015) sobre su exclusión del sistema educativo y del mercado laboral. De forma más concreta, se identifica que los jóvenes dejaron de estudiar de manera prematura puesto que lo hacen principalmente entre los 15 y 17 años, abandonando en los cursos de Educación secundaria Obligatoria de primera etapa, por lo cual, sin haber alcanzado la titulación correspondiente. Coincide con los tramos de edad descritos por la Organización de Naciones Unidas (2014), por la Organización de Cooperación para el Desarrollo Económico (2014), por la Organización Internacional del Trabajo (2015), y con Cartmel (2000).

Segunda: Se observa que la familia ejerce una escasa influencia sobre estos jóvenes para que acudan a los programas de inserción socio laboral, y es que la relación entre NiNi y su familia, resulta bastante compleja en muchas ocasiones. Por otro lado, las amistades o grupo de iguales que no estén implicados en actividades formativas, aún establecen menores relaciones de influencia hacia los NiNi. Coincidimos con las aportaciones de Bivand (2004) sobre la relación del NiNi con la familia y sus grupos de iguales, y las dificultades que se encuentran en su entorno de referencia.

Tercera: Se produce una relación favorable con los programas de inserción. Encontramos mayor implicación en su propio proceso formativo, en el alumnado del grupo experimental que, a su vez, coincide con el que lleva más tiempo y mantiene una asistencia diaria más asidua.

Cuarta: Resulta preocupante que los jóvenes NiNi presentan una autoestima dañada. Aquí coincidimos especialmente con Manassero y Vázquez (1995), Brooks (1998), Steers (2000), y Edcoms (2007), sobre el daño con sufre el NiNi en su autoestima. A pesar de ello, al realizar al respecto un análisis comparativo entre los grupos, se aprecia una mejora generalizada de la autoestima, confirmando así la hipótesis de investigación, enlazando aquí con la relación positiva que existe entre autoestima y un continuo en el rendimiento académico que ya expuso Acuña (2013).

Quinta: Se constata también que, los programas de los centros de inserción socio laboral en los que participan los jóvenes NINI, son capaces de reducir el miedo a la evaluación negativa. Además, se produce una correlación importante entre una mayor autoestima y un menor miedo a la evaluación negativa en estos jóvenes. Observamos pues una conexión ya descrita por Bolívar (2006), o por los hallazgos establecidos por Sims y otros (2001), o por Golden y otros (2008) al respecto. Como afirma Stone y otros (2000), SI mejora la autoestima, mejoramos el miedo a la evaluación negativa. En función de las conclusiones descritas, podemos afirmar que se ha cumplido la hipótesis de investigación: Los programas de los centros 
de inserción sociolaboral de jóvenes NiNi (que ni estudiaban ni trabajaban) son capaces de fortalecer la autoestima y reducir el miedo a la evaluación negativa.

Para finalizar entendemos que sería recomendable que, mediante los programas de inserción socio laboral, los jóvenes NINI, puedan diseñar y desarrollar un proyecto de vida, mediante el cual se incremente su autoestima, la formación en valores, desarrollo de habilidades, actitudes y herramientas, orientado al desarrollo individual de cada uno, potenciando sus capacidades, favoreciendo el proceso de aprendizaje, de tal modo que puedan alcanzar su realización personal, facilitando también que puedan afrontar los retos de un futuro que se presenta exigente antes los cambios e incertidumbres producidos en la sociedad actual en relación a los estudios y el mercado laboral.

\section{Conclusiones}

Esta investigación presenta un valor añadido para la sociedad como predictor de dos variables que afectan a jóvenes en situación de NiNi: autoestima y miedo a la evaluación negativa. Los jóvenes de nuestra sociedad se están viendo castigados por las crisis económicas y sociales durante su desarrollo por lo que resulta fundamental otorgar una validez científica a las variables que se ponen en marcha cuando entran a formar parte de un programa de inserción sociolaboral para así orientar la intervención hacia el fortalecimiento de la autoestima y la disminución del miedo a la evaluación negativa que tanto impide el desarrollo personal y social de los jóvenes que ni estudian ni trabajan.

En cuanto a las limitaciones del estudio, desde la autocrítica podemos identificar la necesidad de diseñar investigaciones más tendentes a la longitudinalidad y al seguimiento de casos de los participantes de la muestra, realizándoles un acompañamiento y apoyo en el tiempo que les sirva de orientación para favorecer su inserción sociolaboral. No debemos olvidar que detrás de todos y cada uno de los encuestados hay personas, con sus circunstancias, características y situaciones que les rodean teniendo todos en común el objetivo de insertarse en la vida social y laboral que les permita el desarrollo personal y en sociedad. Por otra parte, este estudio presenta grandes fortalezas: se trata de una investigación innovadora que relaciona dos variables no estudiadas en el colectivo de jóvenes en situación de NiNi hasta la actualidad. Además, la muestra es representativa de dos barrios que presenta colectivos vulnerables de las ciudades de Sevilla y Málaga, destacando por el número de jóvenes en riesgo de exclusión social. Destaca la implicación de los jóvenes en proporcionar información veraz y el compromiso con la investigación, lo que genera en los investigadores una necesidad de devolver los resultados para continuar mejorando y reforzando los aspectos positivos de este tipo de programas de inserción sociolaboral.

Respecto a las futuras líneas de investigación, estarán dirigidas al estudio de aquellas variables presentes en el proceso de inserción sociolaboral de los jóvenes en nuestra sociedad, primando siempre el favorecimiento de su desarrollo personal y social.

\section{Agradecimientos}

A los centros de inserción sociolaboral Don Bosco, situados en el Polígono Sur de Sevilla y en el barrio de La Trinidad de Málaga.

\section{Referencias}

Acuña, J. (2013). Autoestima y rendimiento académico de los estudiantes del X ciclo 2012 - II de la escuela académica profesional de educación primaria y problemas de aprendizaje de la Universidad Nacional José Faustino Sánchez CarriónHuacho. Universidad Nacional José Faustino Sánchez Carrión.

Archer, L., Halsall, A., Hollingworth, S. y Mendick, H. (2005). Dropping out and drifting away: An Investigation of Factors Affecting Inner-City Pupils' Identities, Aspirations and Post-16 Routes. Institute for Policy Studies In Education.

Bivand, P. (2004). NEET figures continue to rise. Working Brief, 150, 17-19. 
Bolívar, R. (2006). La autoestima se construye constantemente y a lo largo de toda la vida. AUTOR.

Brooks, R. (1998). Staying or leaving? NFER.

Cartmel, F. (2000). Structured chameleons: matching the environment (factors influencing the school to work transition). Youth and Policy, 68, 19-63.

Department for Children, Schools and Families. (2008). Reducing the number ofyoung people not in education, employment or training by 2013. DCSF.

Díaz-Rodríguez, R. P. (2020). Efectos de la autoestima y la seguridad emocional en el sistema familiar sobre el estilo motivacional para el estudio de la Economía. Estudios sobre Educación, 38, 145-167.

https://doi.org/10.15581/004.38.145-167

Edcoms (2009). Participation project desk research. DfES Research Report RW1O2. DFES.

Fergusson, R. (2004). Discourses of exclusion: Reconceptualising participation amongst young people, Journal of Social Policy, 33(2), 289-320. https://doi.org/10.1017/S0047279403007451

Golden, S., Spielhofer, T., Sims, D., Aiston, S. y O’Donnell, L. (2002). Re-engaging the Hardest-to-helpyoung people: The role of the neighbourhood support fund. DFES.

INE (2020). Encuesta de población activa. INE.

Manassero, A. y Vázquez, A. (1995). Dimensionalidad de las causas percibidas en situaciones de éxito y fracaso escolar. Revista de Psicología Social, 10(2), 235-255. https://doi.org/10.1174/021347495763811009

Morales, J.M. (2015). La respuesta comunitaria y española a la realidad de los ninis: Los grandes damnificados por la crisis. Laborum.

Murillo, J. y Salazar, M. A. (2019). La autoestima, la extraversión y el resentimiento como variables predictoras del bienestar subjetivo. Universitas Psychologica, 18(1), art 3. https://doi.org/10.11144/Javeriana.upsy18-1.aerv

Negrete, R. y Leyva, G. (2013). Los NiNis en México: Una aproximación crítica a su medición. Revista Internacional de Estadística y Geografía, 4(1), 90-121.

Rennison, J., Maguire, S., Middleton, S. y Ashworth, K. (2005). Young people not in education, employment or training: Evidence from the education maintenance allowance pilots database. DFES

Rubin, A. y Babbie, E. R. (2005). Research methods for social work. Thomson.

Ruiz-Mosquera, A. C. (2020). Trabajo social en las aulas: Orientación al trabajo mediante las TIC en formación profesional. En M. Nieto Cabrera, M. Vázquez- Fernández y C. Nieto-Morales (Coords.), Atención en la práctica a las personas con necesidad de oportunidades (pp. 195-208). Dykinson. https://doi.org/10.2307/j.ctv17hm88h.14

Ruiz, A.C., Alvarez, J. C. y Palma, M. (2019). NiNis: Juventudy exclusión social. Laborum.

Ruiz-Mosquera, A. C. y Arredondo, R. (2020). Intervención con menores expulsados de clase: Trabajo social educativo. En M. Nieto Cabrera, M. Vázquez- Fernández y C. Nieto-Morales (Coords.), Atención en la práctica a las personas con necesidad de oportunidades (pp. 173-182). Dykinson. https://doi.org/10.2307/j.ctv17hm88h.12

Rosenberg, M. (1965). La autoimagen del adolescente y la sociedad. Paidós.

Sims, D., Nelson, J., Golden, S. y Spielhofer, T. (2001). Young people's experiences of the learning gateway. DFEE.

Spielhofer, T., Walker, W., Gagg, K., Schagen, S. y O'donnell, S. (2007). Raising the participation age in education and training to 18: Review of existing evidence of the benefits and challenges. DCSF.

Steers, R. (2000). A background to youth disaffection: A review of literature and evaluation findings from work with young people. Community Development Foundation.

Vázquez, J., Jiménez, R. y Vázquez-Morejón, R. (2004). Escala de autoestima de Rosenberg: Fiabilidad y validez en población clínica española. Apuntes de Psicología, 22(2), 247-255.

Vázquez-Fernández, M. J. y Ruiz-Mosquera, A. C. (2020). Trabajo social: Instrumento de inserción sociolaboral de colectivos vulnerables. En M. Nieto Cabrera, M. Vázquez- Fernández y C. Nieto-Morales (Coords.), Atención en 
la práctica a las personas con necesidad de oportunidades, (pp. 334-348). Dykinson.

https://doi.org/10.2307/j.ctv17hm88h.21

Watson, D. y Friend, R. (1969). Measurement of social-evaluative anxiety. Journal of Consulting and Clinical Psychology, 33(4), 448-457. https://doi.org/10.1037/h0027806

Zubeidat, I., Salinas, J. M. y Sierra, J. C. (2007). Escala de miedo a la evaluación negativa y escala de evitación y malestar social: Fiabilidad y validez en una muestra de adolescentes españoles. Clínica y Salud, 18(1), 57-81.

\section{Breve CV de los/as autores/as}

\section{Ana Cristina Ruiz-Mosquera}

Doctora en Ciencias Jurídicas y Sociales por la Universidad de Málaga. Graduada en Trabajo Social y posteriormente, Graduada en Psicología por la Universidad de Málaga. Máster en Investigación e Intervención Social y Comunitaria. Máster en Profesorado de Formación Profesional. Durante tres años y medio he dedicado mi investigación a los jóvenes que ni estudian ni trabajan como fenómeno de exclusión social. En 2019 defendí mi tesis doctoral convirtiéndome en la primera doctora del Grado en Trabajo Social por la Universidad de Málaga. Obtuve la mención internacional ya que realicé una estancia predoctoral en la Universidad de Montreal (Canadá). Email: acruizmosquera@uma.es

ORCID ID: https://orcid.org/0000-0003-1920-5834

\section{Ma Josefa Vázquez-Fernández}

Doctora en Ciencias del Trabajo, Licenciada en Ciencias del trabajo, Diplomada en Trabajo Social. Experta en intervención e inserción social y laboral de colectivos en riesgo o situación de exclusión social. Docente en la Facultad de Ciencias Sociales de la Universidad Pablo de Olavide (Sevilla), Departamento de Trabajo Social. Investigadora adscrita al grupo oficial PAI (Plan Andaluz de Investigación) SEJ-452. Secretaría de Universidades Consejería de Innovación, Ciencia y Empresa. Experiencia en la dirección de centros de prevención y rehabilitación de la Ludopatía y otras adicciones y de centros de formación para la inserción laboral. Email: mjvazfer@upo.es

ORCID ID: https://orcid.org/0000-0001-5250-9077

\section{Evaristo Barrera-Algarín3}

Titular de Universidad en el Departamento de Trabajo Social y Servicios Sociales de la Universidad Pablo de Olavide de Sevilla, y Profesor Tutor de la UNED de Sevilla. Trabajador Social. Licenciado en Antropología Social y Cultural. Coordinador del Aula Abierta de Mayores de la Universidad Pablo de Olavide de Sevilla durante sus primeros años y Premio de Investigación 2002 de la Diputación Provincial de Sevilla. Director Académico del Máster en Dirección de Centros Sociales de Personas Mayores. Especializado en Metodología del Trabajo Social. Ha colaborado en la formación de profesionales sociales en los ámbitos públicos y privados. Email: ebaralg@upo.es

ORCID ID: https://orcid.org/0000-0001-8201-9423 